suppress immune-mediated muscle injury are needed. Glucagon-like peptide- 1 (GLP-1) is a peptide hormone with a variety of functions. Although GLP-1 receptor (GLP-1R) agonists have been developed as an anti-diabetic therapy to promote insulin secretion, emerging data suggest that they have pleiotropic actions including anti-inflammatory effects and suppression of muscle wasting ${ }^{2}$. We presumed that GLP-1R agonists have beneficial effect on PM to preserve and recover muscle strength.

Objectives: To examine the effect of a GLP-1R agonist on C protein-induced myositis (CIM), a murine model of polymyositis ${ }^{3}$, in monotherapy or in combination with prednisolone (PSL).

Methods: Muscle specimens of PM patients and CIM were examined with immunohistological staining for the expression of GLP-1R. The therapeutic effect of PF1801 (ImmunoForge), a GLP-1R agonist ( $5 \mathrm{mg} / \mathrm{kg}$ body weight (BW)/day), in monotherapy or in combination with PSL $(20 \mathrm{mg} / \mathrm{kg}$ BW/day) on CIM was examined for grip strength, muscle weight and histological muscle inflammation.

Results: GLP-1R was expressed on the plasma membrane of muscle cells of PM patients and CIM. The expression levels were high in the area where inflammatory infiltrates were observed. The treatment of CIM with PF1801 in monotherapy or in combination with PSL suppressed the CIM-induced decrease in grip strength on day 14. The combination therapy with PF1801 and PSL ameliorated the CIM-induced muscle weight loss in quadriceps, while the monotherapy with PF1801 or PSL did not. The histological analysis of muscle specimens on day 14 of CIM revealed that the muscle inflammation was suppressed by the treatments with PF1801, PSL, or the combination of PF1801 and PSL. None of the mice in the combination therapy group developed histologically evident myositis, while the myositis was observed in $90 \%, 40 \%$ and $40 \%$ of the mice in vehicle treated group, PF1801 treated group, and PSL treated group, respectively. The necrotic area of the muscle in CIM was also reduced in the mice treated with PF1801, PSL, or the combination of PSL and PF1801. The CIM-induced increase in spleen weight was suppressed by PF1801, PSL, or the combination of PSL and PF1801. The additive effect of PSL and PF1801 on the suppression of CIM-induced increase in spleen weight was observed.

Conclusion: PF1801 ameliorated CIM-induced muscle weakness and muscle inflammation in CIM. The combination therapy with PF1801 and PSL ameliorated CIM-induced muscle weight loss. PF1801 could be a novel therapy to recover muscle weakness and to suppress muscle inflammation in PM.

References:

[1] Bronner IM, et al. Ann Rheum Dis. 2006;65:1456-61.

[2] Hong Y, et al. J Cachexia Sarcopenia Muscle. 2019;10:903-18.

[3] Sugihara T, et al. Arthritis Rheum. 2007;56:1304-14.

Disclosure of Interests: Mari Kamiya: None declared, Seon Uk Kim: None declared, Jeong Yeon Kim: None declared, Yeong Wook Song: None declared, Eun Young Lee: None declared, Fumitaka Mizoguchi Grant/research support from: ImmunoForge, Consultant of: ImmunoForge

DOI: 10.1136/annrheumdis-2020-eular.1597

\section{SAT0296 \\ SERUM LEPTIN LEVELS IN SYSTEMIC SCLEROSIS PATIENTS WITH ELECTROCARDIOGRAPHIC ABNORMALITIES}

G. Pellegrino ${ }^{1}$, K. Stefanantoni ${ }^{1}$, F. Facioni ${ }^{1}$, C. Angelelli ${ }^{1}$, A. Gigante ${ }^{2}$, R. Badagliacca ${ }^{1}$, C. D. Vizza ${ }^{1}$, S. Morelli ${ }^{1}$, E. Rosato ${ }^{3}$, V. Riccieri ${ }^{1} .{ }^{1}$ Sapienza University of Rome, Department of Internal Medicine, Anesthesiology and Cardiovascular Sciences, Rome, Italy; ${ }^{2}$ Sapienza University of Rome, Department of Translational and Precision Medicine, Rome, Italy; ${ }^{2}$ Sapienza University of Rome, Department of Translational and Precision Medicine, Rome, Italy

Background: Electrocardiographic (ECG) abnormalities are described in 25-75\% Systemic Sclerosis (SSc) cases and they are associated with other systemic manifestations as well as with a worse prognosis. There is an increasing need for clinical and laboratory biomarkers to ameliorate the diagnostic and therapeutic approaches to patients with ECG abnormalities, due to their actual low sensitivity and specificity. Adipokines are circulating proteins that appear dysregulated in SSc and leptin in particular is synthesized in response to inflammatory conditions and seems to play a proinflammatory and pro-fibrotic action in SSc. Interesting, many studies in the last years have underlined its role in the cardiac remodeling mechanisms and in the development of cardiac fibrosis in other chronic diseases.

Objectives: Aim of our study is to evaluate the role of leptin in the development of cardiac rhythm disorders (CRD) during SSc. Furthermore, by the analysis of the clinical and demographical parameters of our SSc patients, we tried to define other possible features associated with increased serum leptin concentration.
Methods: We included eighty-five SSc patients, fulfilling the $2013 \mathrm{ACR} /$ EULAR classification criteria, attending the Regional Rare Disease Center of Policlinico Umberto I of Rome. Fifty presented significant CRD at non-invasive diagnostic techniques (12 Lead ECG, 24-hour Holter ECG). Demographic, clinical, conventional cardiovascular risk factors were examined instrumental and laboratory assessments were obtained, together with ECG recordings. Thirty-five SSc patients without pathologic finding at ECG traces matched for demographic and clinical features, were recruited as the control group. In all cases, after obtaining written informed consent, blood samples were taken to measure serum levels of leptin using an ELISA assay (Life Technologies-Italia).

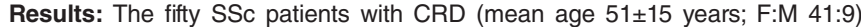
had pulmonary fibrosis (PF) in 32 cases $(64 \%)$ and a BMI $>25 \mathrm{Kg} / \mathrm{m}^{2}$ in $22(44 \%)$ while in the control group of thirty-five SSc patients (mean age $49 \pm 16$ years; F:M 33:2) PF was found in $15(43 \%)$ and a $\mathrm{BMI}>25 \mathrm{Kg} / \mathrm{m}^{2}$ in 9 (35\%); We detected significantly higher median values of serum leptin in SSc patients with CRD compared to the control group (12027 pg/ml IQR 12314 versus $6392 \mathrm{pg} / \mathrm{ml}$ IQR 7103; p 0,0009). Additionally, SSc patients with a BMl> $25 \mathrm{~kg} / \mathrm{m}^{2}$ (31 cases) as well as those with PF (47 cases) showed a significantly higher median serum leptin levels compared to those with $\mathrm{BMI}<25 \mathrm{~kg} / \mathrm{m}^{2}$ (13161 pg/ml IQR 13610 versus $8187 \mathrm{pg} /$ $\mathrm{ml}$ IQR 8255; $p$ 0,0008) and those without PF (11740 pg/ml IQR 11940 versus $7616 \mathrm{pg} / \mathrm{ml}$ IQR $7855 ;$ p 0,0079).

Conclusion: To our knowledge this is the first report on high serum levels of leptin in SSc patients with CRD that also confirms its increase in those cases with a $\mathrm{BMI}>25 \mathrm{~kg} / \mathrm{m}^{2}$ and with PF, according to scientific literature data. The role of leptin in the pathogenesis of SSc remains unclear although it is already known its involvement in the development of cardiac fibrosis during other chronic diseases. On the basis of these results we speculate on leptin involvement in the pathogenesis of CRD during SSc, although further studies are needed with larger cohort of patients.

References:

[1] Vacca A et al. Rheumatology, 2014

[2] Tyndall AJ et al. Ann Rheum Dis, 2010

[3] Muresan L et al. Iran J Pub Health, 2017

[4] Sanna T et al. Indian Pacing Electrophysiol J, 2009

[5] Riccieri V et al. Clin Exp Rheumatol, 2011

[6] Żółkiewicz J et al. Arch Dermatol Res, 2019

[7] Huby AC et al. Circulation, 2015

[8] Shulze PC et al. Clin Chim Acta, 2005

[9] Van de Hoogen F et al. Arthritis Rheum, 2013

[10] Gui $X$ et al. Biochem Biophys Res Commun, 2018

Disclosure of Interests: Greta Pellegrino: None declared, Katia Stefanantoni Consultant of: Italfarmaco

Boehringer Ingelheim, Fausta Facioni: None declared, Carlotta Angelelli: None declared, Antonietta Gigante: None declared, Roberto Badagliacca: None declared, Carmine Dario Vizza: None declared, Sergio Morelli: None declared Edoardo Rosato: None declared, Valeria Riccieri: None declared DOI: 10.1136/annrheumdis-2020-eular.3390

\section{SAT0297 \\ DIFFERENTIAL PHENOTYPES OF DISEASE-SPECIFIC AUTO-REACTIVE B CELL RESPONSES IN PATIENTS WITH SYSTEMIC SCLEROSIS}

C. Wortel ${ }^{1}$, N. Van Leeuwen2, M. Boonstra ${ }^{1}$, R. Toes ${ }^{1}$, T. Huizinga ${ }^{1}$, J. De Vries-Bouwstra ${ }^{1}$, H. U. Scherer ${ }^{1}$. 'Leiden University Medical Center, Leiden, Netherlands; ${ }^{1}$ Leiden University Medical Center, Leiden, Netherlands

Background: Systemic Sclerosis (SSc) carries the highest mortality burden among the rheumatic diseases. $>95 \%$ of SSc patients harbor autoantibodies. Anti-topoisomerase antibodies (ATA) and anti-centromere antibodies (ACA) are most prevalent, mutually exclusive in individual patients, associate with distinct disease phenotypes and predict disease. Whether and how these auto-reactive $B$ cell responses contribute to disease, however, is currently unclear.

Objectives: To delineate phenotypic and functional characteristics of anti-topoisomerase and anti-centromere specific B cell responses in individual patients with SSc.

Methods: Peripheral blood mononuclear cells (PBMC) obtained from ATA- and ACA-positive SSc patients were cultured without stimulation or in the presence of CD40L-expressing fibroblasts, IL-21 and BAFF. In addition, PBMC were depleted of circulating plasmablasts (CD19+CD20-CD27++) by fluorescence activated cell sorting (FACS), and isolated plasmablasts were cultured separately. ATAand ACA-IgG and -IgA were measured in culture supernatants by ELISA. B cell 
subsets were defined by flow cytometry. Healthy donors and patients with rheumatoid arthritis served as controls.

Results: We observed that ATA- and ACA-positive SSc patients harbour circulating B cells that secrete either ATA-IgG or ACA-IgG upon stimulation, depending on their serotype. In addition, we noted spontaneous secretion of ATA-IgG and, more remarkably, extensive secretion of ATA-IgA in ATA-positive patients. This degree of spontaneous, antigen-specific IgA secretion was specific for the ATA response in ATA-positive patients, while spontaneous ACA-IgA secretion was undetectable in the ACA-positive patient group. FACS experiments showed that spontaneously ATA-IgA secreting B cells were primarily present in the plasmablast compartment.

Conclusion: Our findings demonstrate that ATA-positive SSc patients harbour an activated ATA-IgG and ATA-IgA B cell response, as indicated by the spontaneous secretion of both ATA isotypes by circulating plasmablasts. Remarkably, the ACA B cell response was far less active and lacked the active IgA component which suggests a difference in the triggers driving these autoreactive $B$ cell responses in patients. Moreover, the remarkable ATA-IgA secretion points towards a potential mucosal origin of the ATA response.

Disclosure of Interests: Corrie Wortel: None declared, Nina van Leeuwen: None declared, Maaike Boonstra: None declared, Rene Toes: None declared, Thomas Huizinga Grant/research support from: Ablynx, Bristol-Myers Squibb, Roche, Sanofi, Consultant of: Ablynx, Bristol-Myers Squibb, Roche, Sanofi, Jeska de Vries-Bouwstra: None declared, Hans Ulrich Scherer Grant/research support from: Bristol Myers Squibb, Sanofi, Pfizer, Speakers bureau: Pfizer, Lilly, Roche, Abbvie

DOI: 10.1136/annrheumdis-2020-eular.6049

\section{SAT0298 IS INTERLEUKIN 6 A FACTOR OF FIBROGENESIS IN DERMAL FIBROBLASTS?}

A. S. Siebuhr ${ }^{1}$, P. Juhl ${ }^{1}$, M. Karsdal ${ }^{2}$, A. C. Bay-Jensen ${ }^{1} .{ }^{1}$ Nordic Bioscience, ImmunoScience, Herlev, Denmark; ${ }^{2}$ Nordic Bioscience, R\&D, Herlev, Denmark

Background: Interleukin 6 (IL-6) is known to have both pro- and anti-inflammatory properties, depending on the receptor activation. The classical IL-6 signaling via the membrane bound receptor is mainly anti-inflammatory, whereas signaling through the soluble receptor (sIL-6R) is pro-inflammatory/pro-fibrotic. However, the direct fibrotic effect of IL-6 stimulation on dermal fibroblasts is unknown.

Objectives: We investigated the fibrotic effect of IL-6 + sIL-6R in a dermal fibroblast model and assessed fibrosis by neo-epitope biomarkers of extracellular matrix proteins.

Methods: Primary healthy human dermal fibroblasts were grown for up to 17 days in DMEM medium with $0.4 \%$ fetal calf serum, ficoll (to produce a crowded environment) and ascorbic acid. IL-6 [1-90 nM]+SIL-6R [0.1-9 nM] alone or in combination with TGF $\beta[1 \mathrm{nM}$ ] were tested in three different donors. TGF $\beta$ [1 nM], PDGF-AB [3 nM] and non-stimulated cells (w/o) were used as controls. Tocilizumab (TCZ) with TGF $\beta$ + IL-6 + sIL-6R stimulation was tested in one donor. Collagen type I, $\mathrm{III}$ and VI formation (PRO-C1, PRO-C3 and PRO-C6) and fibronectin (FBN-C) were evaluated by validated ELISAs (Nordic Bioscience). Western blot analysis investigated signal cascades. Gene expression of selected ECM proteins was analyzed. Statistical analyses included One-way and 2-way ANOVA and area under the curve analysis.

Results: formation by the end of the culture period. The fibronectin and collagen type VI signal were consistent between the three tested donors, whereas the formation of type III collagen was only increased in one donor, but in several trials. Type I collagen formation was unchanged by IL- 6 + sIL-6R stimulation. The gene expression of type I collagen was induced by IL-6 + sIL-6R. Western blot analysis validated trans-signaling by the IL-6+sIL-6R stimulation as expected.

IL-6 + sIL-6R stimulation in combination with TGF $\beta$ decreased fibronectin levels compared to TGF $\beta$ alone but did not reach the level of unstimulated fibroblasts. The formation of collagen type IV was generally unchanged with IL-6 + sIL-6R + TGF $\beta$ compared to TGF $\beta$ alone. Collagen type I and III formation was more scattered in the signals when IL- 6 + SIL-6R was in combination with TGF $\beta$, as the biomarker level could be either decreased or increased compared to TGF $\beta$ alone. In two studies the type I collagen level was synergistic increased by IL- 6 + sIL-6R + TGF $\beta$, whereas another study found the level to be decreased compared to TGF $\beta$ alone. The gene expression of fibronectin and type I collagen was increased with TGF $\beta+$ IL-6+sIL-6R compared to TGF $\beta$ alone.

Inhibition of IL-6R by TCZ in combination with IL-6 + sIL-6R did only decrease the fibronectin level with the lowest TCZ concentration $(p=0.03)$. TCZ alone decreased the fibronectin level in a dose-dependent manner (One-way ANOVA $\mathrm{p}=0.0002$ )

Conclusion: We investigated the fibrotic response of dermal fibroblasts to IL-6 + sIL-6R stimulation. IL-6 modulated the fibronectin level and modulated the collagen type III formation level in a somewhat dose-dependent manner. In combination with TGF $\beta$, IL- 6 decreased collagen type I and IV formation and fibronectin. However, in this study inhibition of IL-6R by TCZ did not change the fibrotic response of the dermal fibroblasts. This study indicated that IL-6 did not induce collagen formation in dermal fibroblasts, except type III collagen formation with high IL-6 concentration.

\section{Figure:}

Flbronectin remodeling by IL-6

in TGF $\beta$ stimulated dermal fibroblasts

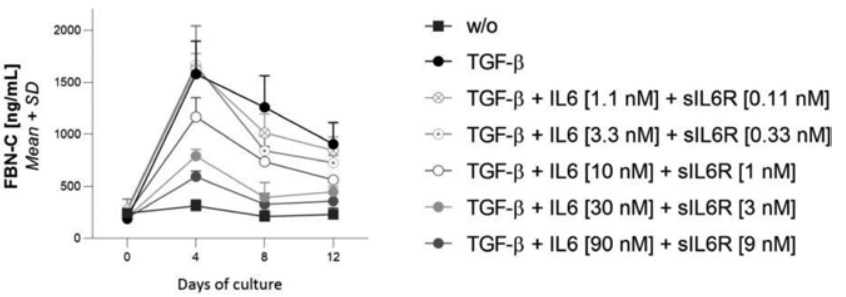

Gene expression in SiaJ

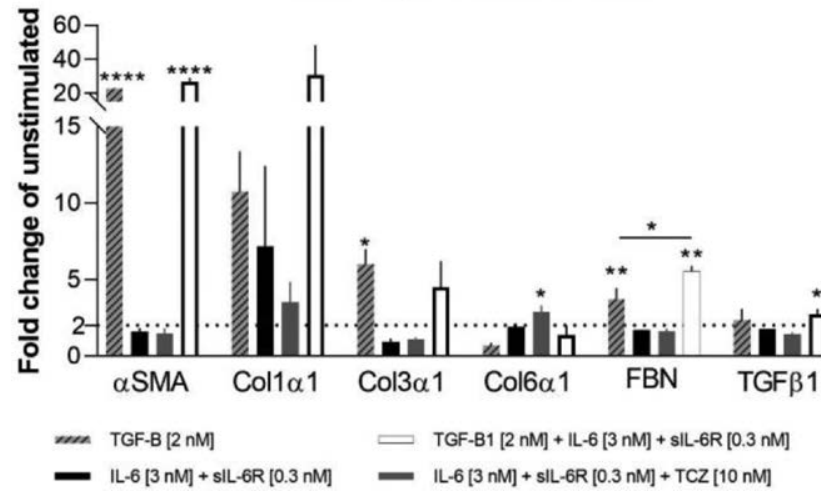

Disclosure of Interests: Anne Sofie Siebuhr Employee of: Nordic Bioscience, Pernille Juhl Employee of: Nordic Bioscience, Morten Karsdal Shareholde of: Nordic Bioscience A/S., Employee of: Full time employee at Nordic Bioscience A/S., Anne-Christine Bay-Jensen Shareholder of: Nordic Bioscience A/S Employee of: Full time employee at Nordic Bioscience A/S.

DOI: 10.1136/annrheumdis-2020-eular.4061

\section{SAT0299 PROLIFERATION, MIGRATION AND CONTRACTION ARE DIFFERENT BETWEEN TGFB AND PDGF STIMULATED DERMAL FIBROBLASTS}

A. S. Siebuhr ${ }^{1}$, S. F. Madsen ${ }^{1}$, M. Karsdal ${ }^{2}$, A. C. Bay-Jensen ${ }^{1}$, P. Juhl' ${ }^{1}{ }^{1}$ Nordic Bioscience, ImmunoScience, Herlev, Denmark; ${ }^{2}$ Nordic Bioscience, R\&D, Herlev, Denmark

Background: Systemic sclerosis has vascular, inflammatory and fibrotic components, which may be associated with different growth factors and cytokines. Platelet derived growth factor (PDGF) is associated with the vasculature whereas tumor necrosis factor beta (TGF $\beta$ ) is associated with inflammation and fibrosis. We have developed a fibroblast model system of dermal fibrosis for anti-fibrotic drugs testing, but the effect of the fibroblasts mechanistic properties are unknown.

Objectives: We investigated different mechanical capacities of PDGF and TGF $\beta$ treated human healthy dermal fibroblasts in the SiaJ setting

Methods: Primary human healthy dermal fibroblasts were grown in DMEM medium containing $0.4 \%$ fetal calf serum, ficoll (to produce a crowded environment) and ascorbic acid for up to 17 days. A wound was induced by scratching the cells at $0,1,3$ or 7 days after treatment initiation. Wound closure was followed for 3 days. Contraction capacity was tested by creating gels of human fibroblasts produced collagens containing dermal fibroblasts and contraction was assessed at day 2 by calculating the percentage of gel size to total well size. Collagen type I, III and VI formation (PRO-C1, PRO-C3 and PRO-C6) and fibronectin (FBN-C) 